\title{
Cavitation modeling using compressible Navier-Stokes and Korteweg equations
}

\author{
T. Ait Ali $^{1}$, S. Khelladi ${ }^{1}$, L. Ramirez ${ }^{2} \&$ X. Nogueira ${ }^{2}$ \\ ${ }^{1}$ DynFluid Laboratory, Arts et Métiers ParisTech, France \\ ${ }^{2}$ GMNI, Universidade da Coruña, Spain
}

\begin{abstract}
A high accurate finite volume method based on the Moving Least Squares (MLS) approximation is used for the resolution of compressible Navier-Stokes and Korteweg (NSK) equation in order to simulate the inception of bubble cavitation, its detachment and its collapse on the upper surface of a 2D NACA 0015 hydrofoil. To properly simulate these phenomena, we use the homogeneous equilibrium model (HEM) whose main assumption is that we consider a diffuse liquid-vapor interface with thickness. To take account of the surface tension force through the NSK equations, we need to compute a third derivative of density.

In this study, we present evidence for the effect of surface tension on the maximum pressure reached during the collapse of a cavitation bubble. It was noticed that increasing the surface tension inevitably leads to an increase in the pressure of the collapse. Beyond a certain value of $\lambda$ (which depends on velocity, incidence and other parameters) the cavitation bubbles are more difficult to separate from the main cavity that forms on the upper surface of the hydrofoil. Keywords: cavitation, homogeneous model, Moving Least Squares, surface tension, Korteweg tensor, hydrodynamic, Tait equation of state for water.
\end{abstract}

\section{Introduction}

Cavitation is a complex phenomenon which is due to the turbulence that reigns above and coexistence, in a very small space, of two phases (liquid and its vapor) whose physical properties are radically different. To best simulate this phenomenon one uses homogeneous equilibrium model (HEM) [1] associated to compressible Navier-Stokes. Using an equation of state for each fluid phase: Modified Tait equation of state for the liquid, gas equation of state for the vapor 
and the saturation pressure for the mixture located in the liquid-vapor interface. The set of equations is solved with a high order finite volume scheme based on Moving Least Squares (MLS) [2] reconstruction with a numerical flux adapted, as well, for high and very low Mach numbers. The proposed approach allows the use of unstructured meshes for complex geometries. The thermodynamic effects are taken into account through the resolution of the energy equation with subtracting the latent heat during vaporization.

To take into account the effects of the surface tension, Korteweg source term is used as developed by Diehl [3].

\section{Homogeneous equilibrium model for cavitation flow}

Following Koop [1], homogeneous flow theory provides the simplest description for analyzing multi-phase or multi-component flows. the equilibrium cavitation model adopted is the one developed by Stewart and Wendroff [4] and Schmidt et al. [5]. This physical model is based on the assumption that the two-phase flow regime can be described as a homogeneous mixture of vapor and liquid that remains in thermodynamic and mechanical equilibrium. This implies that in the mixture locally the vapor and liquid have equal temperature, pressure and velocity. This assumption is based on the belief that differences in these three potential variables will promote momentum, energy, and mass transfer between the phases rapidly enough so that equilibrium is reached. Throughout the computational field, a single system of conservation equations are used to describe the flow which is considered compressible. To close the system, different equations of state can be used. we chose to use an equation of state for each phase. Equation of Tait for Liquid and ideal gas for water vapor and a saturation pressure for the mixture phase. The void fraction of vapor $\alpha$ is used to determine the state of the fluid according its density. It is defined by equation (1). This dimensionless parameter is very important because it allows to know the proportion of vapor in the mixture.

$$
\alpha=\frac{V_{v}}{V}=\frac{\rho-\rho_{l, s a t}(T)}{\rho_{v, s a t}(T)-\rho_{l, s a t}(T)}
$$

\subsection{Formulation of the Navier-Stokes and Korteweg equations}

The governing equations are the compressible 2D Navier-Stokes equations in conservation form here for Cartesian coordinates as

$$
\frac{\partial \mathbf{U}}{\partial t}+\frac{\partial \mathbf{F}_{\mathbf{x}}(\mathbf{U})}{\partial x}+\frac{\partial \mathbf{F}_{\mathbf{y}}(\mathbf{U})}{\partial y}=S
$$

The integral formulation of equation (2) is used. Applying the divergence theorem, for each cell on 2D we have:

$$
\frac{\partial}{\partial t} \iint_{\Omega} \mathbf{U} d \Omega+\int_{\partial \Omega}\left(\mathbf{F}_{\mathbf{x}} \cdot \mathbf{n}_{\mathbf{x}}+\mathbf{F}_{\mathbf{y}} \cdot \mathbf{n}_{\mathbf{y}}\right) d l=\iint_{\Omega} \mathbf{S} d \Omega
$$


Here,

$$
\mathbf{U}=(\rho \rho u \rho v \rho E)^{T} \quad \text { and } \quad \mathbf{S}=\left(0 S_{x} S_{y} 0\right)^{T}
$$

$\mathbf{U}$ is the vector of conserved variables, $\mathbf{S}$ is the source term of Korteweg (see section 2.2 equation (15)) and $\left(F_{x}, F_{y}\right)$ the components of flux vector which can be divided into convective and diffusive flux. Those are given as

$$
\begin{gathered}
\mathbf{F}_{\mathbf{x}}=\mathbf{F}_{\mathbf{x}}{ }^{C}+\mathbf{F}_{\mathbf{x}}{ }^{V} \\
\mathbf{F}_{\mathbf{y}}=\mathbf{F}_{\mathbf{y}}{ }^{C}+\mathbf{F}_{\mathbf{y}}{ }^{V} \\
\mathbf{F}_{\mathbf{x}}{ }^{C}=\left(\begin{array}{c}
\rho u \\
\rho u^{2}+p \\
\rho u v \\
(\rho E+p) u
\end{array}\right), \quad \mathbf{F}_{\mathbf{x}}{ }^{V}=\left(\begin{array}{c}
0 \\
\tau_{x x} \\
\tau_{x y} \\
\rho v \\
\tau_{x y}-q_{x}
\end{array}\right) \\
\mathbf{F}_{\mathbf{y}}{ }^{C}=\left(\begin{array}{c}
u \tau_{x x} \\
\rho v u \\
\rho v^{2}+p \\
(\rho E+p) v
\end{array}\right), \quad \mathbf{F}_{\mathbf{y}}{ }^{V}=\left(\begin{array}{c}
0 \\
\tau_{x y} \\
\tau_{y y} \\
u \tau_{x y}+v \tau_{y y}-q_{y}
\end{array}\right)
\end{gathered}
$$

The viscous tensor is defined as

$$
\begin{gathered}
\tau_{x x}=2 \mu\left(\frac{\partial u}{\partial x}-\frac{2}{3}\left(\frac{\partial u}{\partial x}+\frac{\partial v}{\partial y}\right)\right) \\
\tau_{y y}=2 \mu\left(\frac{\partial v}{\partial y}-\frac{2}{3}\left(\frac{\partial u}{\partial x}+\frac{\partial v}{\partial y}\right)\right) \\
\tau_{x y}=\mu\left(\frac{\partial u}{\partial y}+\frac{\partial v}{\partial x}\right)
\end{gathered}
$$

\subsection{Surface tension model (the Korteweg tensor)}

The HEM model suppose that the density varies continuously through the interface. In this case, Korteweg term is more appropriate to describe surface tension. It is introduced as a source term in momentum Navier-Stokes equations, see (12).

$$
\frac{\partial \rho u}{\partial t}+\nabla \cdot\left(\rho u u^{T}\right)+\nabla p=\nabla \cdot(\tau)+\nabla \cdot(\mathbf{K})
$$

$\mathrm{K}$ is called Korteweg tensor. It is divided into two parts. The Symmetric parts induces a normal strength opposite to the density gradient. The second part induces 
tangential strength relative to density gradient.

$$
K=\lambda\left[\left(\rho \Delta \rho+\frac{1}{2}|\nabla \rho|^{2}\right) \boldsymbol{I}-\nabla \rho \nabla \rho^{T}\right]
$$

For the Korteweg tensor we have this useful identity

$$
\nabla \cdot K=\lambda \rho \nabla \Delta \rho
$$

By developing the expression (14) in 2D, we find density derivatives of order three (see equation (15)). To correctly estimate these derivatives we need to use high order of spacial discretization (see section 3.2).

$$
S_{x}=\lambda \rho\left(\frac{\partial^{3} \rho}{\partial x^{3}}+\frac{\partial^{3} \rho}{\partial x \partial y^{2}}\right) \quad S_{y}=\lambda \rho\left(\frac{\partial^{3} \rho}{\partial y \partial x^{2}}+\frac{\partial^{3} \rho}{\partial y^{3}}\right)
$$

\subsubsection{Estimation of capillary coefficient $\lambda$}

The real thickness of liquid-vapor interface is too small to be captured. For water at $295 \mathrm{~K}$ the thickness of the interface (noted $\mathrm{h}$ ) is around $0.9 \mathrm{~nm}$. For usual flow that we study, mesh size we need to capture the interface is huge. the solution proposed in this paper is to consider an artificial thickness for the interface (noted $h$ ) which has the same order as the size of one cell of the mesh and from there we determine a numerical value of $\lambda$ such as the surface tension forces remains the same. We use the equation (16) developed by Jamet et al. [6] to compute numerical $\lambda$ using the artificial thickness $\mathrm{h}$ and the real surface tension $\sigma$.

$$
\lambda=\frac{3}{2} \frac{\sigma h}{\left(\rho_{l}-\rho_{v}\right)^{2}}
$$

\subsection{Equations of state}

For closure of the system of equations it is necessary to adopt equations of state that describe each of the three possible states: the liquid state, the vapor state and the mixture state.

For realistic simulation of phase transition and to preserve the hyperbolic nature of the system of governing equations we use three different EOS. In the following the liquid phase is denoted by subscript $l$, the vapor phase by subscript $v$, mixture phase by subscript $m$ and saturation conditions by subscript sat. The speed of sound $c\left[\mathrm{~ms}^{-1}\right]$ and dynamic viscosity $\mu[\mathrm{kg} / \mathrm{ms}]$ will be defined for each state in table 1.

For water vapor $\gamma_{v}=1.32 . L_{v}(T 0)=2.753 \cdot 10^{6} \mathrm{~J} \mathrm{~kg}^{-1} \mathrm{~K}^{-1}$ represents the latent heat of vaporization, $e_{l 0}=617 \mathrm{~J} \mathrm{~kg}^{-1}$ is the internal energy at this reference temperature $T=T_{0}=273.15 \mathrm{~K}$. $C_{v v}$ the specific vapor heat at constant volume. $\mu_{0}$ is the viscosity at temperature $T_{0} . R_{v}$ is the specific constant of water vapor. $K_{0}$ and $N$ are constants which depend on the fluid. For water, $K_{0}=3.310^{8} \mathrm{~Pa}$, $N=7.15, A=2.414 \cdot 10^{-5} \mathrm{~Pa}, B=247.8 \mathrm{~K}$ and $C=140 \mathrm{~K} . C_{v l}$ is the liquid specific heat at constant volume. 
Table 1: Pressure, energy, viscosity and sound velocity for the three EOS.

\begin{tabular}{|l|c|}
\hline \multicolumn{2}{|c|}{ Liquid } \\
\hline Pressure & $p_{l}=K_{0}\left[\left(\frac{\rho}{\rho_{l, \text { sat }}(T)}\right)^{N}-1\right]+P_{\text {sat }}\left(T_{l}\right)$ \\
\hline $\begin{array}{l}\text { Internal } \\
\text { Energy }\end{array}$ & $e_{l}=C_{v l}\left(T_{l}-T_{0}\right)+e_{l 0}$ \\
\hline Viscosity & $\mu_{l}=A \cdot 10^{B / T_{l}-C}$ \\
\hline $\begin{array}{l}\text { Sound } \\
\text { Velocity }\end{array}$ & $c_{0}^{2}=\left(N\left(p-p_{\text {sat }}(T)+K_{0}\right) / \rho+p /\left(\rho^{2} C_{v} l\right)\left[\partial p_{\text {sat }}(T) / \partial T-\right.\right.$ \\
& $\left(\partial \rho_{l, s a t}(T) / \partial T\right)\left(N\left(p-p_{\text {sat }}(T)+K_{0}\right) / \rho_{l, s a t}(T)\right]$ \\
\hline
\end{tabular}

\begin{tabular}{|l|c|}
\hline \multicolumn{2}{|c|}{ Vapor } \\
\hline Pressure & $p_{v}=\left(\gamma_{v}-1\right) \rho_{v} e_{v}$ \\
\hline $\begin{array}{l}\text { Internal } \\
\text { Energy }\end{array}$ & $e_{v}=C_{v v}\left(T_{v}-T_{0}\right)+L_{v}\left(T_{0}\right)+e_{l 0}$ \\
\hline Viscosity & $\mu_{v}=\mu_{0}\left(\frac{T_{v}}{T_{0}}\right)^{\left(\frac{3}{2}\right)} \frac{T_{0}+s}{T_{v}+s}$ \\
\hline $\begin{array}{l}\text { Sound } \\
\text { Velocity }\end{array}$ & $c_{v}=\sqrt{\gamma_{v} R_{v} T_{v}}$ \\
\hline
\end{tabular}

\begin{tabular}{|l|c|}
\hline \multicolumn{2}{|c|}{ Mixture } \\
\hline Pressure & $p=p_{\text {sat }}(T)$ \\
\hline $\begin{array}{l}\text { Internal } \\
\text { Energy }\end{array}$ & $\rho e=\alpha \rho_{v, \text { sat }}(T) e_{v}(T)+(1-\alpha) \rho_{l, \text { sat }}(T) e_{l}(T)$ \\
\hline Viscosity & $\mu_{m}=\alpha \mu_{v}+(1-\alpha) \mu_{l}$ \\
\hline $\begin{array}{l}\text { Sound } \\
\text { Velocity }\end{array}$ & $1 / \rho c_{m}^{2}=\alpha /\left(\rho_{v, \text { sat }}(T) c_{v}^{2}\right)+(1-\alpha) /\left(\rho_{l, \text { sat }}(T) c_{l}^{2}\right)$ \\
\hline
\end{tabular}




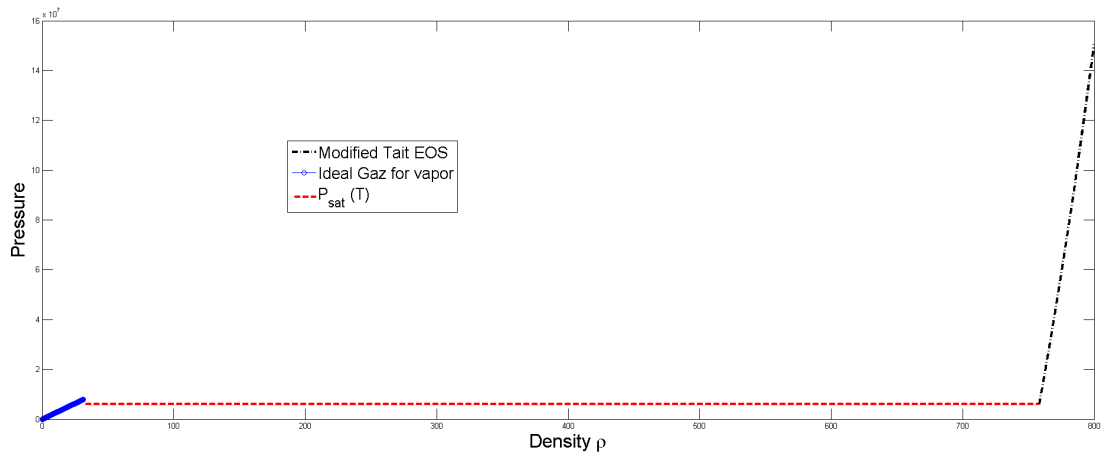

Figure 1: The three equation of state at temperature $550 \mathrm{~K}$ that describes the three phases.

We show in figure 1 the system of three equation of state that we use. We choose to plot it at $T=550 \mathrm{~K}$ to see the vapor part which is to small at low temperature.

\section{Numerical method}

The HEM equations presented in equation (3) are solved employing an unstructured grid, Moving Least-Squares based of high order finite-volume method developed in [7] and [8]. Dividing the physical domain in control volumes $\Omega_{i}$ with boundary $\partial \Omega_{i}$. Equation (3) can be considered for each control volume and the transitory term of the equation is rewritten as

$$
\frac{\partial}{\partial t} \iiint_{\Omega_{i}} \mathbf{U} d \Omega_{i}=\Omega \frac{\partial \bar{U}_{i}}{\partial t}
$$

Where $\bar{U}_{i}=\iiint_{\Omega_{i}} \mathbf{U}(x, y) d \Omega_{i}$ is the averaged value of the flow variables in volume control $\Omega_{i}$ Flux are supposed constant through each edge of the cell. Equation (3) becomes

$$
\frac{\partial \bar{U}_{i}}{\partial t}=\frac{-1}{\Omega}\left[\sum_{k=1}^{N}\left(F^{C}(U)-F^{D}(U)\right) \cdot n_{k} S_{k}\right]=\frac{-1}{\Omega} R
$$

$F^{C}$ is the convective flux and $F^{D}$ the diffusive flux. $N$ is the number of cell edges. $S_{k}$ the length of the $k$ th edge and $R$ is the residual.

\subsection{Variable reconstruction}

The FV-MLS method starts from a high-order and highly regular representation of the solution, obtained by means of Moving Least-Squares approximation. the 
mean idea is to approximate $U(x)$, at a given point $x$, through a weighted leastsquares fitting of $U(x)$ in a neighborhood of $x$ as

$$
\left.U(x) \approx \tilde{U}_{[} x_{I}\right](x)=\sum_{i=1}^{m} p_{i}(x) \alpha_{i}=p^{T}(x) \alpha\left(x_{i}\right)
$$

$p^{T}$ is an $m$-dimensional polynomial basis and $\alpha\left(x_{i}\right)$ is a set of parameters to be determined, such that they minimize the following error functional

$$
J\left(\alpha\left(x_{i}\right)\right)=\int_{\Gamma_{x_{I}}} W_{\left[x_{i}\right]}(y)\left[U(y)-p^{T}(y) \alpha\left(x_{I}\right)\right]^{2} d y
$$

Being $W_{\left[x_{i}\right]}$ the exponential kernel function which is defined as

$$
W\left(x_{j}-x_{I}, \kappa\right)=\frac{e^{-\left(\frac{s}{c}\right)^{2}}-e^{-\left(\frac{d}{c}\right)^{2}}}{1-e^{-\left(\frac{d}{c}\right)^{2}}}
$$

with $s=\left|x_{j}-x_{I}\right|, d=\max \left(\left|x-x_{I}\right|\right), c=\frac{d}{2 \kappa}, x_{I}$ is the center of the stencil and $\kappa$ is a shape parameter. Shape function are used to express variable reconstruction from node value and given as

$$
N_{\left[x_{I}\right]}(x)^{T}=\mathbf{p}\left(\frac{x-x_{I}}{h}\right)^{T} \cdot\left(P_{\Omega_{x_{I}}} \cdot W_{\left[x_{I}\right]}(x) \cdot P_{\Omega_{x_{I}}}^{T}\right)^{-1} \cdot P_{\Omega_{x_{I}}} \cdot W_{\left[x_{I}\right]}(x)
$$

where $\mathrm{h}$ is the smooth parameter. So variables can be approximated by shape functions as

$$
\tilde{U}_{\left[x_{I}\right]}(x)=N_{\left[x_{I}\right]}(x)^{T} U_{\Omega_{x_{I}}}=\sum_{j} N_{X_{I}^{(j)}} U_{j}
$$

with $U_{\Omega_{x_{I}}}$ the variable vector $\mathrm{U}$ of each cell of the stencil.

\subsection{Estimation of partial variables derivatives}

Using Taylor expansion of $U$ at the vicinity of (I) and equation (23) the variable derivatives reconstruction is given as

$$
\frac{\partial^{\alpha} U}{\partial x^{\alpha-\beta} \partial y^{\beta}}\left(., x_{I}\right)=\sum_{j} \frac{\partial^{\alpha} N_{x_{I}^{(j)}}}{\partial x^{\alpha-\beta} \partial y^{\beta}}\left(x_{I}\right) \cdot U_{j}
$$

We remark that the approximation is written in terms of the MLS "shape functions" $N_{x_{I}^{(j)}}$, that are depending on the grid. So, in fixed grids they will be computed only once. the MLS approximation will be used to compute the derivatives needed for the reconstruction of variables at quadrature points at cell interfaces. 


\subsection{Flux discretization}

The main idea behind the higher-order formulation of the FV-MLS is the use of reconstructed variables of a given order of accuracy into the numerical flux, to achieve the desired order of the scheme. Flux must be estimated at cell interfaces. The extrapolated left (+) and right (-) states at each edge integration point leads the solution to be discontinuous. An approximate Riemann solver is used in order to fit the solution. For the convective flux the SLAU [9] (Simple Low dissipation AUSM) scheme is used.

Using high order scheme to solve non linear equations induces appearance of numerical oscillations which causes instabilities. The higher-order accuracy are obtained by introducing a Venkatakrishnan flux-limited dissipation [10,11]. The viscous terms are discretized as simple average between right and left value of the normal viscous flux.

\section{Results}

\subsection{Numerical simulation of bubble}

To see the effect of the Korteweg term in the pressure. Let us see the case of a vapor bubble of radius $R=0.284 \mathrm{~m}$ surrounded by water. For initial conditions, we put pressure equal to saturation pressure, temperature $T=295 \mathrm{~K}$ and the initial density $\rho$ is expressed in the equation (25). The computational domain is $\Omega=\left[\begin{array}{ll}-0.5 & 0.5\end{array}\right] \times\left[\begin{array}{ll}-0.5 & 0.5\end{array}\right]$

$$
\rho_{0}=500.95+499.05\left(\tanh \left(\frac{(d-R) L_{0}}{2 h}\right)\right)
$$

We use the equation (25) to have continuous variation of the density through the interface (see more in [12]). This give us the dashed curve in figure 2(b). Here $d=\sqrt{x^{2}+y^{2}}(m), L_{0}=1 \mathrm{~m}$ is the length of the domain and $h=(1 / 64) \mathrm{m}$ is the length of one cell.

When we set $\lambda=0$ we see in the figure 2(b) that the bubble remains steady. When we set $\lambda=0.15625 \mathrm{~m}^{7} \mathrm{~kg}^{-1} \mathrm{~s}^{-2}$ we get a compression in a bubble vapor due to the Korteweg forces represented in figure 2(d). We notice that the increasing of the bubble pressure is followed by an increasing in the density and temperature, and finally the bubble collapses.

In this example we used intentionally high values of $\lambda$ to show how the Korteweg term influences the pressure inside a vapor bubble.

\subsection{Cavitating flow around an hydrofoil}

We consider the Sauer test case (see [13]) which describe a turbulent cavitating flow around a NACA0015 hydrofoil at angle of attack $\tilde{\alpha}=6$. We use an unstructured grid with 9578 triangular elements. The velocity $V_{\infty}=12 \mathrm{~m} / \mathrm{s}$, 


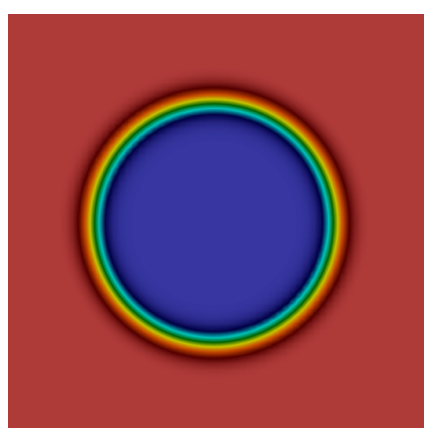

(a) Bubble shape

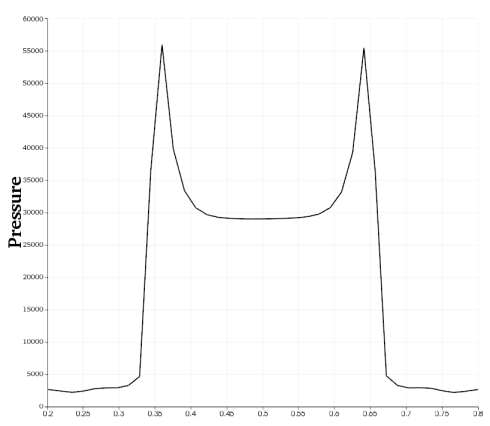

(c) Pressure variation trough bubble

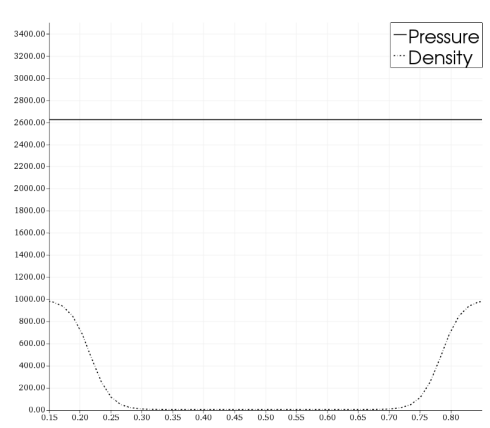

(b) Pressure variation trough bubble

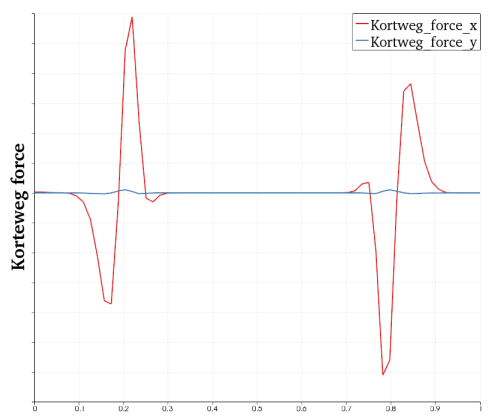

(d) Korteweg force in the interface

Figure 2: Comparison between vapor bubble with $\lambda=0$ (a,b) and $\lambda=$ $0.15625 \mathrm{~m}^{7} \mathrm{~kg}^{-1} \mathrm{~s}^{-2}$ (c, d).

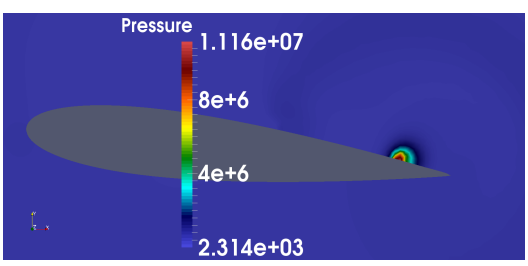

(a)

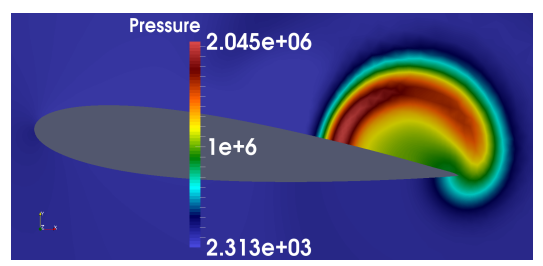

(b)

Figure 3: (a) Maximum pressure reached in the collapse (Pa). (b) Pressure distribution after bubble collapse $(\mathrm{Pa})$.

initial pressure $p_{\infty}=50000 \mathrm{~Pa}$, temperature $T_{\infty}=293 \mathrm{~K}$. This correspond to a cavitation number of $\Gamma=0.66$. We use SLAU scheme [9] for numerical flux, with explicit temporal discretization which has a fixed time step of $10^{-8} \mathrm{~s}$. The chord of the hydrofoil $c=0.15 \mathrm{~m}$ and the domain is an $\mathrm{O}$ mesh which radius $R=5 \mathrm{~m} . \lambda$ is computed from $\sigma$ using equation (16) where $L_{0}=$ chord $(0.15 \mathrm{~m})$ and $h=$ average of $\sqrt{\text { cell area }}$ around the hydrofoil. 


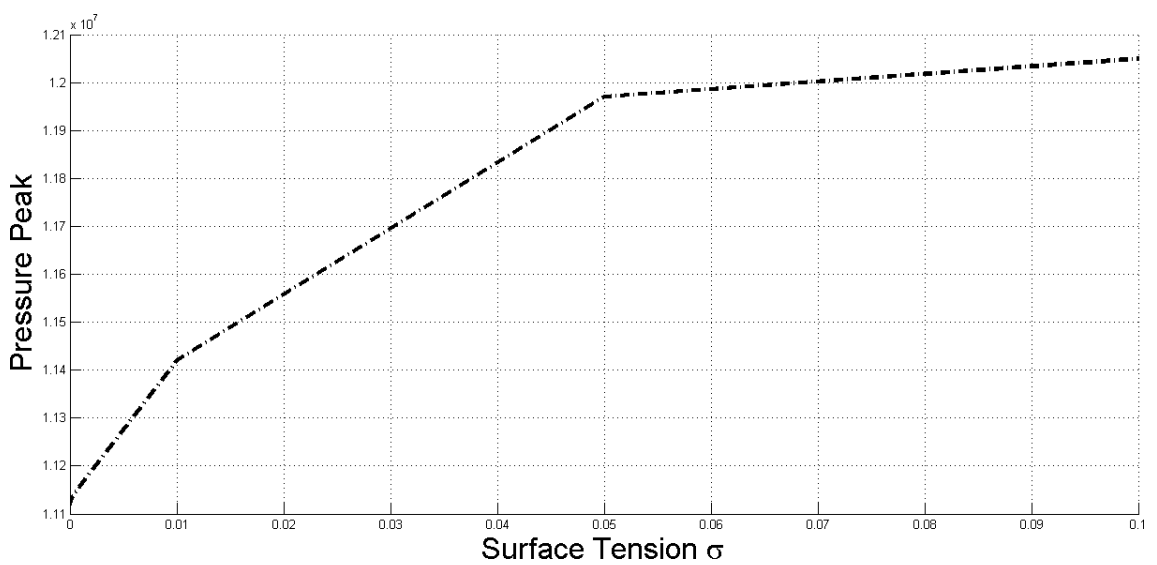

Figure 4: Evolution of pressure peak $(\mathrm{Pa})$ with the surface tension $\sigma(\mathrm{N} / \mathrm{m})$.

Let us focus on the collapse of the first bubble of vapor that occurs around the fourth millisecond. To be sure to capture the maximum pressure we make a backup every $10^{-6} \mathrm{~s}$. For different value of the surface tension $\sigma$ we trace maximum pressure showed in figure 4.

The graph 4 shows that the increase in surface tension leads to an increase of the pressure peak. The effect of surface tension start to be significant at $\sigma=10^{-2} \mathrm{~N} / \mathrm{m}$. On the other hand the effect of surface tension appears in the final stage of collapse when the bubble reached a size of the order of a millimeter or less.

\section{Conclusion}

In this paper we presented a cavitation model that is homogeneous equilibrium model. To take into account the surface tension we used the Navier-Stokes and Korteweg compressible unsteady. A high order Finite Volume method based on Moving Least Squares approximation is used with unstructured mesh. An MLS reconstruction is used for the spatial discretization of variables and their derivatives. we used an MLS reconstruction in order to determine the third derivatives of density in the Korteweg term. The first case to test validated the digital schematic for the consideration of the surface tension. Trough the first computation, we see how the surface tension forces directly influence the pressure and the temperature in vapor bubble by generating surface force in the interface. A second series of calculation was performed to determine the effect of the consideration of the surface tension on the collapse of the cavitation bubbles on the upper surface of a hydrofoil. We noticed that an increase of surface tension causes a direct increase in the peak pressure reached during the collapse. this means that for the study of cavitation including the collapse it is best not to neglect the surface tension. 


\section{References}

[1] Koop, A.H., Numerical simulation of unsteady three-dimensional sheet cavitation. University of Twente, 2008.

[2] Khelladi, S., Nogueira, X., Bakir, F. \& Colominas, I., Toward a higher order unsteady finite volume solver based on reproducing kernel methods. Computer Methods in Applied Mechanics and Engineering, 200(29), pp. 2348-2362, 2011.

[3] Diehl, D., Higher order schemes for simulation of compressible liquid-vapor flows with phase change. Ph.D. thesis, Universitätsbibliothek Freiburg, 2007.

[4] Bruce Stewart, H. \& Wendroff, B., Two-phase flow: models and methods. Journal of Computational Physics, 56(3), pp. 363-409, 1984.

[5] Schmidt, S.J., Sezal, I.H. \& Schnerr, G.H., Compressible simulation of highspeed hydrodynamics with phase change. pp. 5-8, 2006.

[6] Jamet, D., Lebaigue, O., Coutris, N. \& Delhaye, J., The second gradient method for the direct numerical simulation of liquid-vapor flows with phase change. Journal of Computational Physics, 169(2), pp. 624-651, 2001.

[7] Cueto-Felgueroso, L., Colominas, I., Nogueira, X., Navarrina, F. \& Casteleiro, M., Finite volume solvers and moving least-squares approximations for the compressible Navier-Stokes equations on unstructured grids. Computer Methods in Applied Mechanics and Engineering, 196(45), pp. 4712-4736, 2007.

[8] Nogueira, X., Khelladi, S., Cueto-Felgueroso, L., Bakirt, F., Colominas, I. \& Gómez, H., Implicit large-eddy simulation with a moving least squaresbased finite volume method. IOP Conference Series: Materials Science and Engineering, IOP Publishing, volume 10, p. 012235, 2010.

[9] Kitamura, K., Fujimoto, K., Shima, E. \& Wang, Z., Performance of lowdissipation euler fluxes and preconditioned implicit schemes in low speeds. AIAA Paper, 1272, pp. 4-7, 2010.

[10] Venkatakrishnan, V., On the accuracy of limiters and convergence to steady state solutions. AIAA Paper, 93-0880, 1993.

[11] Venkatakrishnan, V., Convergence to steady state solutions of the euler equations on unstructured grids with limiters. Journal of Computational Physics, 118(1), pp. 120-130, 1995.

[12] Gomez, H., Hughes, T.J., Nogueira, X. \& Calo, V.M., Isogeometric analysis of the isothermal navier-stokes-korteweg equations. Computer Methods in Applied Mechanics and Engineering, 199(25), pp. 1828-1840, 2010.

[13] Sauer, J. \& Schnerr, G., Unsteady cavitating flow-a new cavitation model based on modified front capturing method and bubble dynamics. ASME Fluids Engineering Division Summer Meeting. Boston, Massachusetts, USA, 2000. 